\title{
Deleuze y Rancière: la política como resistencia
}

\author{
Deleuze and Rancière: politics as resistance
}

\author{
Leticia Arancibia Martínez ${ }^{[a]}$, Teresa Montealegre Barba ${ }^{[b] *}$ \\ [a] Pontificia Universidad Católica de Valparaíso, Valparaíso, Chile \\ [b] Université Paris VIII Vincennes-Saint-Denis, Saint-Denis, França
}

\section{Resumen}

El presente artículo intenta indagar en el estatus político de la filosofía a partir de una lectura de Gilles Deleuze y Jacques Rancière. Este vínculo nos parece pertinente para comprender o dar un nuevo enfoque a la crisis actual de lo político, en la que pareciera no haber alternativa al presentarse como un dispositivo que reproduce la desigualdad y descontento social (política institucional, política de partidos). La elaboración de esta investigación, intenta mostrar que la crisis existente tiene como parte del problema su concepción de política misma. Es por ello que la pregunta ¿qué es realmente la política?, es fundamental para pensar nuestra actualidad. Por lo anterior, la relevancia de la concepción de política en ambos autores, reside en su radicalización que permite evidenciar a toda política institucional como policial. La política siempre surge y refiere a un Afuera que se resiste a ser apresado por un sistema, institución y toda lógica normativa. Lo político es resistencia pura, devenir en proceso. A través del examen del vínculo

* LAM: Doctora en Sciences Politiques et Sociales, e-mail: leticia.arancibia@gmail.com TMB: Licenciada en Filosofía,e-mail: maria.montealegre@ug.uchile.cl 
entre filosofía y política (la llamada filosofía política), se propone una aproximación a una comprensión de lo político entendido como resistencia elaborada por ambos autores, vínculo que logra dar cuenta de la potencialidad de la política y del carácter dual de la filosofía. Esta dualidad es dada por su carácter creacional donde por un lado, tiene un rasgo emancipatorio, y por otro, un rasgo fundacional. Con respecto a lo anterior, se trabajará principalmente a partir de una lectura de ¿Qué es la filosofía? y Conversaciones, de Deleuze, haciendo aproximaciones a la obra de Rancière a partir de El desacuerdo y Aux bords du politique, principalmente.

Palabras clave: Política. Devenir. Filosofía. Deleuze. Rancière.

\section{Abstract}

This article intends to inquire into the Political Status of philosophy from a reading of Gilles Deleuze and Jacques Rancière. This link appears to be pertinent in order to comprehend or give a new scope to the actual crisis of Politics when it seems to be no alternative to Present seen as a device that reproduces de inequality and social discontent (instituted politics, politics of parties). This survey tries to expose that the actual crisis has in the concept of politics itself, an important part of the problem. Thus, the question What politics really is? is of fundamental importance to think our current time. For that, the relevance of the concept of Politics in both authors resides in its radicalization that puts in evidence all political institutionalization as a form of police. Politics always refers and surges as an Outside that resists to be caught by a system, institution or regulatory logic. The political itself is pure resistance, a development in process. Through the examination of the strange marriage between Philosophy and Politics (the so called Political Philosophy), an approach to a comprehension of the concept "political" understood as a resistance in both authors, is proposed. A link that shows the potentiality of politics and a dual character of Philosophy, duality given by its creational character that is both foundational and emancipatory at the same time. Therefore, the work will develop from the readings of Deleuze's What is Philosophy? and Conversations, making approaches to the work of Rancière, mainly The disagreement and Aux bords du politique.

Keywords: Politics. Becoming. Philosophy. Deleuze. Rancière. 


\section{Introducción}

Una serie de acontecimientos vertiginosos irrumpen en la política contemporánea cuestionando, poniendo en tensión o reforzando un cierto orden social que aparece como el depositario del poder político. Algunas veces ese cuestionamiento de las instituciones, desencadena en algo nuevo, o bien da paso a mecanismos de reemplazo, y captura del poder en la política contingente. Ante las discusiones, pero también los procesos de categorización o resemantización que acontecen en la escena contingente, la filosofía aparece enfrentada ante el problema de su relación con la política.

La preocupación por la política se ve enfrentada al devenir de la filosofía, interrogada respecto de sus alcances y límites. ¿Cómo se enfrenta el problema de la relación entre la política y la filosofía, considerando al mismo tiempo su devenir? ¿Por qué la pregunta sobre la filosofía debería excluir o escindirse de la política? ¿Es posible la filosofía política?

Se propone una reflexión sobre el alcance de la filosofía en Deleuze, desde una lectura de la política, marcado por el ejercicio de situarse dentro de lo que podríamos llamar un "plano de inmanencia rancièreano". Tomando del pensamiento político de Jacques Rancière el problema de la constitución de un sujeto político y cómo es que éste se forma, se considerarán elementos del pensar político de Deleuze, dando valor al devenir de la filosofía como emancipación, para, a partir de este diálogo entre ambos autores, entre ambos planos, dar cuenta de la actitud común de su pensamiento: una filosofía de la resistencia, en medio del conflicto que comporta la política y su constitución paradójica.

La discusión se desarrollará en tres apartados. Nos embarcaremos, en una primera parte, en la concepción de filosofía que trata Deleuze en conjunto con Guattari, en su libro ¿Qué es la filosofía?, entrada que creemos, nos permite dar con una posible definición de lo político en dicho autor. En segundo lugar, se planteará la interrogante de si es la filosofía, según su definición, un acto verdaderamente político, vinculándolo, con la noción de lo político en Rancière. Y en una tercera 
parte, trataremos la relación entre devenir y política, tomando como fuente principal la entrevista de Toni Negri a Deleuze. Finalmente a modo de síntesis, se desarrollará en una última parte los paralelos pertinentes entre ambos autores.

Intentaremos, a través de este itinerario comprender y establecer lo propiamente político en dichos autores, entendido como resistencia.

La obra de Deleuze ha estado fuertemente marcada por la insistencia en demarcarse de aquella visión dogmática del pensar, de todo esencialismo o metafísica platónica que ha dominado el pensamiento occidental. Desde el comienzo de su obra se plantea la necesidad de construir una «nueva imagen del pensamiento». Se rechaza la idea que ha mantenido la tradición del pensamiento, de lo verdadero como fundamento, como aquella acción que capta la realidad como tal $^{1}$. La filosofía de Deleuze, rescata el acontecimiento como intento de liberar lo infinito, como intento de develar a la realidad como caos, como desbordamiento que escapa a toda categoría que la presente como esencial. Su filosofía está marcada por el devenir de la existencia. La necesidad de construir una nueva imagen del pensamiento, es el intento de escapar del pensamiento dogmático que nos impide pensar verdaderamente, o dicho de otro modo, que nos obliga a pensar de una determinada forma.

Es a partir de aquí que se afirma que la obra deleuziana está atravesada por un carácter político, a pesar de que no hace referencia explícita ni propone una definición a dicho concepto. Para desarrollar esta idea es fundamental, primero, comprender qué es entendido

\footnotetext{
El rechazo a la visión dogmática del pensamiento, idea característica de la filosofía francesa contemporánea, se apoya en la idea de comprender al concepto como algo vivo, como creación, como un proceso. Esta idea es fundamental para entender el tratamiento filosófico de autores como Rancière y Deleuze, especialmente en conceptos como lo político. Badiou (2014) en el mismo sentido, plantea la necesidad de no oponer más la existencia a la vida: "Mostrar que el concepto es algo vivo, una creación, un proceso y un acontecimiento, y que por tal razón no está separado de la existencia (p.18).
} 
como político en este autor, y creemos que a través del tratamiento de su noción de filosofía, podremos comprenderlo.

En su obra ¿Qué es la filosofía? (1991), responde a la pregunta negativamente señalando que ésta no es ni contemplación, ni reflexión, ni comunicación, ya que esos aspectos suponen una realidad externa al pensamiento que la filosofía debe captar y luego comunicar. La filosofía, para Deleuze, es el acto de inventar, de formar, de fabricar conceptos, lo que supone una desnaturalización, un cuestionamiento del concepto como algo dado que se manifiesta por sí mismo, cuando en cambio, es el resultado de una creación. Cabe la pregunta ¿qué es, entonces, un concepto?

Deleuze señala que todo concepto está constituido por una serie de elementos o componentes que lo definen.; Los conceptos son un todo fragmentario que no se ajustan unos con otros, puesto que sus bordes no coinciden. Y sin embargo resuenan "y la filosofía que los crea presenta siempre un Todo poderoso, no fragmentado [...] que incluye a todos en un único y mismo plano" (2001, p. 39). Este plano de consistencia que genera la ilusión de un Todo único, es el «plano de inmanencia». De modo que la filosofía no solo debe crear conceptos, sino, también establecer un campo de inmanencia. El plano es el depósito de los acontecimientos conceptuales, es el horizonte absoluto e independiente de cualquier observador, es lo que garantiza el contacto de los conceptos (2001, p. 41). El plano de inmanencia no es un concepto pensable ni pensado, sino que es la imagen del pensamiento, la imagen de lo que significa pensar. "El plano es por lo tanto objeto de una especificación infinita, que hace que tan solo parezca ser el Uno-Todo en cada caso especificado por la selección de movimiento" (2001, p. 43). Es por esto que el plano de inmanencia tiene que ser considerado prefilosófico, no conceptual, a pesar que la filosofía la suponga: "El concepto es el inicio de la filosofía, pero el plano es su instauración" (2001, p. 45).

La filosofía se enfrenta al caos a través de la creación de conceptos y el establecimiento de un plano de inmanencia que parcela una sección del caos, permitiendo dar un sentido a lo infinito, una forma a la existencia que se caracteriza por su ausencia de forma 
(2001, p. 46). Sin embargo, el gran problema de la filosofía radica en darle consistencia, sin perder lo infinito; por lo que existen una multiplicidad de planos, ya que cada uno de ellos retiene solo algunos movimientos del caos, de lo infinito, a pesar de que se tenga la ilusión de que cada plano es un Uno-Todo que describe la realidad como único modo de ser. La filosofía es devenir y coexistencia de los diversos planos que entran en diálogo unos con otros, que a medida que revocan planos anteriores, evocan a otros más antiguos. Por ello - afirma Deleuze - aquellos que no replanteen la imagen del pensamiento, que no den cuenta de lo dado como ilusión y que no creen nuevos planos de inmanencia, no pueden ser llamados filósofos (2001, p. 54). La filosofía es creación y no hay creación de la nada, por lo que siempre supone la disolución de lo anterior y la reconfiguración de un nuevo modo de percibir.

Ahora bien, bajo esta definición de filosofía surgen preguntas: ¿no es la desnaturalización y la creación un acto de resistencia a lo dado? ¿y no es la resistencia, precisamente, un acto político?

\section{II.}

Con lo anterior tendríamos una posible definición de lo político como acto de creación y resistencia al orden normativo de la sociedad. Deleuze en la entrevista publicada en la revista Cinemá de 1985, señala que "Toda creación tiene un valor político y un contenido político" (1996, p. 86). El acto de creación supone un orden anterior que fue reestructurado, recortado o incluso echado abajo, por lo tanto supone una actitud de resistencia. En este sentido Deleuze afirma que el concepto posee en sí mismo una fuerza crítica de liberación del flujo infinito del pensamiento, y por lo tanto, una fuerza política. Crear conceptos es resistir.

Ahora bien, ¿esto significa una homologación entre filosofía y política? ¿es la filosofía, entonces, siempre un acto político?

Es cierto que hasta este punto la definición de la filosofía y de lo político parecieran ser una misma cosa. Sin embargo, recordemos 
que la filosofía consiste también en la nueva instauración de un orden y con ello, en un nuevo apresamiento del devenir en un sistema que otorga sentido al caos. Podríamos identificar, de este modo, dos momentos en el acto filosófico:

1) Un primer momento de resistencia como liberación del flujo del caos, como liberación del devenir. Este es, para nosotros, el momento propiamente político.

2) Y un segundo momento de creación en sí, donde se vuelve a establecer o fijar un nuevo orden de cosas, un plano de inmanencia, y con ello el aparataje conceptual que interpreta los acontecimientos y sus movimientos infinitos.

De este modo, lo propiamente político en la filosofía correspondería a este primer momento de creación como resistencia al plano de inmanencia dominante; mientras que en el segundo momento la filosofía se establece como creación en sí, como el nuevo orden que redefine las regiones del plano, que crea sentido y por lo tanto, que impone su adecuación ${ }^{2}$.

Para explicar lo anterior, nos parece pertinente el análisis de Badiou en su artículo ¿Existe algo así como una política deleuziana? publicado en la revista Cité en el 2009. Aquí señala lo siguiente:

La «máxima política» en Deleuze enuncia que «el devenir es más importante que la historia». [...] Se debe crear algo nuevo. La sociedad de control es solamente la organización de la prohibición del devenir. Así, la política - la verdadera política - es la liberación del deseo y el devenir. Sin embargo, la creación misma no es política. [...] la política es una máxima de creación y no una creación en sí (2009, p. 17).

2 Cabe señalar que para Deleuze la creación de un nuevo plano de inmanencia, la creación filosófica, no debe ser entendida como una creación ex nihilo, sino más bien, como un nuevo orden, un nuevo recorte de la realidad, una nueva parcelación del caos. 
Con lo anterior tenemos que la filosofía tiene como máxima lo político, es decir, es movida por un principio político: el devenir y la liberación del devenir. Sin embargo, la filosofía no es política, porque ella es creación en sí misma, es instauración de un nuevo estado de cosas que vuelve a fijar y estructurar un nuevo modo de percibir la realidad. La filosofía tiene un momento originario que es fundamentalmente político y subversión del fundamento, pero luego construye fundamento, dándole a su momento político clausura, dominando nuevamente el devenir. Pues lo político es pura resistencia, liberación del devenir que es prohibido por la sociedad de control.

Es aquí donde la concepción rancièreana de lo político comienza a resonar, entrando en diálogo con el problema planteado. Antes de introducirnos en su tratamiento del problema de la relación filosofía y política, es preciso hacer un breve esbozo de qué es lo que entiende Rancière por política.

Para Rancière, uno de los conceptos fundamentales para comprender lo político, es el de «Reparto de lo sensible», el que permite dar cuenta de la experiencia (sensible) de una realidad que nos parece dada. Experiencia que se encuentra bajo una estructura determinada, normada y susceptible de reproducción por una lógica que permite su validación y mantenimiento en el tiempo (RANCIÈRE, 2009, p. 9). El reparto de lo sensible es el régimen que establece una estructura como única forma de comprender la realidad, el que define los límites entre aquello que forma parte en ese reparto (ya sean los nacionales, los propietarios, los dirigentes, o bien la razón, el buen gusto, la autoridad, la tradición) y aquello que queda excluido (el extranjero, el pobre, el desheredado, o bien el desorden, la ruptura, la crítica). Esta operación donde la política aparece reducida a su dimensión normativa, como una forma de "organización de la prohibición del devenir", fija identidades, clausura la creación de nuevos sentidos que se salen del reparto establecido y desvanece el plano de la inmanencia que permite la crítica y la resistencia. 
La lógica que reproduce y establece que ese determinado modo de sentir y percibir se mantenga en el tiempo, está más interesada de asegurar el funcionamiento de aquello que es parte de un cierto orden, que de abrirse a las posibilidades del devenir, esto es lo que el autor llamará policía (RANCIÈRE, 2006, p. 17). La policía "incorpora a la comunidad en la asimilación de sus leyes a unas maneras de vivir, al principio de respiración de un cuerpo vivo" (RANCIÈRE, 2012, p. 87). Por otra parte, tenemos la lógica emancipatoria, aquel no-lugar, un Afuera que el propio Reparto fija. Esta lógica hace alusión al pueblo o a las llamadas por Deleuze "minorías" (DELEUZE, 1996, p. 241).

Considerando los tres términos: la policía, la emancipación y lo político, lo político consiste en el terreno de encuentro y enfrentamiento entre ambas lógicas. Lo político es dado como revelación contra la lógica policial, como resistencia a un régimen de lo sensible que pretende darse como único modo de ser; por ello significa la apertura hacia el conflicto, que permite la distinción de los planos en juego, evidenciando las exclusiones y los bordes que se fijan para ellas, ofreciendo posibilidades de creación de algo nuevo y diferente. Lo político es liberación del devenir: donde la lógica emancipatoria lucha por desprenderse de lo policial para devenir, para convertirse en algo nuevo.

Retomando la cuestión acerca de la filosofía y su relación con lo político, podemos ver que Rancière hace básicamente el mismo movimiento que señalamos anteriormente en Deleuze. En su libro El Desacuerdo, el francés señala que el extraño encuentro entre filosofía y política expresa una imposibilidad de que la filosofía pueda ser política (2012, p. 11): a pesar de que la filosofía de cuenta de que lo dado por el Reparto, es ilusorio y entre en desacuerdo con aquellas categorías y conceptos que establece ese orden, ella nunca puede ser política, en la medida que siempre vuelve a fijar un nuevo reparto de lo sensible, vuelve a atrapar el devenir de la realidad en un sistema filosófico. Para Rancière, la filosofía, incluso si es filosofía política, opera bajo una lógica policial, ya que establece un Reparto de lo sensible (que podría entenderse como el plano de inmanencia 
de Deleuze), y crea conceptos, define, describe y norma lo social. El riesgo de "Identificar la política con la policía puede significar también identificar la policía con la política, [lo que significaría] construir una imitación de la política", cuando la "naturalidad policial” se perdió (RANCIÈRE, 2012, p. 87). Allí la filosofía enfrenta la tensión, en la que vendría a sostener un orden bajo una "existencia vegetativa" en el régimen policial, en su intento de responder ante la paradoja que plantea la política (que Rancière hará equivalente a la democracia ${ }^{3}$, en tanto no la concibe como un "régimen o un modo de vida social" [sino como] la institución de la política misma" (RANCIÈRE, 2012, p. 128), donde "la democracia propone captar un inconmensurable específico, de una parte de los que no tienen parte como problema a resolver por la filosofía" (RANCIÈRE, 2012, p. 87).

En síntesis, habría en la filosofía para Rancière al igual que para Deleuze, un primer momento político de resistencia y creación, sin embargo, en un segundo momento acabaría determinándola como policía, como normativa, capturando y acotando las posibilidades de creación y devenir, saliendo del plano de inmanencia que "aperturizaba" lo político.

\section{III.}

Lo político en ambos autores es un movimiento que está en constante resurgimiento y disolución, es principalmente cambio, efervescencia emancipatoria. De este modo lo propiamente político es el devenir. Es aquí donde surge el conflicto: el ejercicio filosófico no puede ser estrictamente político, porque el acto de creación en sí implica necesariamente la instauración de un nuevo orden, deteniendo o congelando el movimiento propio de lo político: el devenir.

3 Según Rancière (2011) La democracia es el nombre de una doble ruptura: la separación del nacimiento consigo mismo y del mandamiento consigo mismo. Esta ruptura no es otra cosa que una desnaturalización, una interrupción de la naturalidad de la dominación. De modo que la democracia "antes de ser un «régimen político», la democracia es el régimen de la política" (p. 45) 
Intentaremos profundizar en esta idea a partir de las respuestas que nos brinda Deleuze en la entrevista realizada por Toni Negri en 1990. En ella, Deleuze nos relata su interés en las creaciones colectivas, los procesos de subjetivación y con ello, en el devenir revolucionario, presentándolo como la única alternativa que los hombres tienen de responder a lo intolerable, de resistir a la sociedad de control (1996, p. 238). El pueblo, la minoría, es considerada por el autor como devenir en proceso. Cuando habla de minorías, no refiere a una cuestión cuantitativa, sino un modo de participación en lo social que es representado como falta, que designa a aquellos que no tienen lugar, aquellos que carecen de modelo. Por otro lado, la mayoría es definida por quienes están dentro del modelo establecido como verdadero, al que solo hay que adecuarse.

Es así como las minorías, cuando crean sus modelos (manifiestan su propio reparto de lo sensible) son movidas por su deseo de convertirse en mayoría, como estrategia de supervivencia. El pueblo, como lógica emancipatoria, siempre desea convertirse en policía, siempre desea legitimar su reparto de lo sensible.

Para ambos autores, es el proceso de subjetivación el que permite que un sujeto o una comunidad se manifieste como devenir en lo social. Sin embargo, estos procesos de subjetivación - señala Deleuze - sólo son válidos en la medida que escapen al mismo tiempo de los saberes constituidos y de los poderes dominantes. Es decir, debe ser un proceso de subjetivación y no de identificación, una creación de nuevas categorías y no una adecuación a las categorías ya establecidas. Y agrega lo siguiente: “Aunque ellos se prolonguen en nuevos poderes o provoquen nuevos saberes: tienen en su momento una espontaneidad rebelde" (1996, p. 245).

Nos parece importante la cita anterior, ya que lo político radica en este momento de espontaneidad rebelde, de absoluto devenir. Devela también el fenómeno de suspensión de lo político al dar cuenta de cómo la minoría puede volverse mayoría, de cómo la lógica emancipatoria se convierte en lógica policial, es decir, de cómo la resistencia, lo propiamente político, en el momento que se instaura 
como poder, se convierte en el nuevo núcleo duro que normativiza los espacios.

Lo político se desvanece en el momento que se legitima. Lo fundamentalmente político debe ser entendido, entonces, como proceso, como movimiento, como puro devenir; lo político sucede como interrupción ${ }^{4}$ del sistema establecido (en Rancière) o como liberación del flujo infinito del devenir (en Deleuze).

Todo reparto de lo sensible crea un afuera. Cualquier delimitación crea su propia periferia y por lo tanto su propia condición de posibilidad de lo político, a la vez esto manifiesta que todo reparto es creación de sentido, y por lo tanto, ilusión que parcela el caos. De este modo, cuando la minoría deviene mayoría, vuelve a fijar un Afuera, un no-lugar, volviéndose policial y creando nuevos sectores de resistencia. Es por esta razón que lo político es devenir en proceso, ya que en el momento que la fuerza política se legitima, se frena el devenir, y comienza a brotar en la nueva periferia.

\section{Consideraciones finales}

La filosofía de Deleuze tanto como la de Rancière manifiesta un elemento común: la inquietud por liberar el pensamiento de toda dominación que lo norme o limite. De este modo podríamos caracterizar su filosofía como un pensamiento del afuera, como un pensamiento de la resistencia. Es por ello que creemos que la comprensión de lo político en ambos autores, es fundamental para entender su filosofía.

Para nuestros autores, lo político pone de manifiesto que no existe un solo modo de percibir, de comprender, de sentir. Lo político es el terreno de encuentro de una multiplicidad de regímenes

4 La interrupción da cuenta del carácter temporal de la política en Rancière, de momentos de irrupción del tiempo normal de la dominación: "Hay política cuando la contingencia igualitaria interrumpe como 'libertad' del pueblo al orden natural de las dominaciones, cuando esta interrupción produce un dispositivo específico: una división de la sociedad en partes que no son 'verdaderas' partes; la institución de una parte que se iguala al todo en nombre de una 'propiedad' que es la comunidad de un litigio" (RANCIÈRE, 2012, p. 33). 
de lo sensible y por lo tanto de creación y coexistencia de sentido. De este mismo modo, lo político es la resistencia a toda determinación policial (como diría Rancière), a la vez que manifestación de las minorías como desbordamiento de dichas categorías. Lo político es movimiento, liberación y absoluto devenir, por lo que todo intento de ordenar, definir o sistematizar dicho flujo, anula lo político.

Así, el encuentro problemático de la filosofía con lo político es que a pesar de que ésta actúa en forma revolucionaria y subversiva, en el momento de su consumación se vuelve esencialmente antipolítica. La filosofía política en su intento frustrado de liberar el freno del devenir sólo puede poner nuevas resistencias, nuevos frenos, nuevos repartos de lo sensible, por lo que siempre termina siendo policial, y no política (como desea), es aquí donde reside su destino trágico. La filosofía hace preguntas, y con ellas desnaturaliza, critica y da cuenta del flujo infinito del pensamiento. Sin embargo, siempre termina dando respuesta, paralizando el movimiento infinito del devenir.

¿No resuena esto a la molesta idea de que la filosofía es el arte de hacer preguntas y no dar respuestas?

\section{Referencias}

BADIOU, A. Existe-t-il quelque chose comme une politique deleuzienne? Cités, n. 40, p. 15-20, 2009.

BADIOU, A. La aventura de la filosofía francesa, a partir de 1960 (I. Agoff, Trad.). Santiago: LOM Ediciones, 2014.

DELEUZE, G.; GUATTARI, F. ¿Qué es la filosofía? Trad. Kauf. Barcelona: Anagrama, 2001.

DELEUZE, G.; GUATTARI, F. Mil Mesetas. Capitalismo y ezquisofrenia. (Trad. J. Vasquez Pérez. Valencia: Pre-textos, 2002.

DELEUZE, G. Conversaciones 1972-1990. Trad. J. L. Pardo. Valencia: Pre-textos, 1996. 
RANCIÈRE, J. El desacuerdo. Trad. H. Pons. Buenos Aires: Ediciones Nueva Visión, 2012.

RANCIÈRE, J. Política, policía, democracia. Trad. M. E. Tijoux. Santiago de Chile: LOM Ediciones, 2006.

RANCIÈRE, J. El reparto de lo sensible. Santiago: LOM Ediciones, 2009.

RANCIÈRE, J. Aux bords du politique. París: La Fabrique éditions, 1998.

RANCIÈRE, J. El odio a la democracia. Trad. I. Agoff . Buenos Aires: Amorrortu, 2012.

RANCIÈRE, J. Momentos políticos. Trad. G. Villalba. Buenos Aires: Clave intelectual, 2011.

Recibido: 22/10/2016

Recebido: 22/10/2016

Aprobado: 14/01/2017

Aprovado: 14/01/2017 